\title{
A Guide to Implement Open Data in Public Agencies
}

\author{
Mauricio Solar ${ }^{1}$, Luis Meijueiro ${ }^{2}$, and Fernando Daniels ${ }^{3}$ \\ ${ }^{1}$ Universidad Técnica Federico Santa María, Chile \\ ${ }^{2}$ Fundación CTIC, Spain \\ ${ }^{3}$ Inter-american Organization for Higher Education (OUI-IOHE) \\ mauricio.solar@usm.cl, luis.meijueiro@fundacionctic.org, \\ danielsf@oui-iohe.org
}

\begin{abstract}
This article presents a guide to implement open data in Public Agencies (PAs). The guide is the result of a worldwide proposal's study, of the application of a maturity model to diagnose the situation of PAs in Latin American countries, the opinion of experts in different excellence centers, egovernment authorities, and developers of open data application in the world. The guide is simple and orients decision makers so that PAs following the actions of the guide can see their capacities improved when facing a diagnosis of their institutional maturity in implementation of open data.
\end{abstract}

Keywords: open data, open government data, roadmap, maturity model.

\section{Introduction}

The need to encourage data reuse is a key task, since it allows promoting the capacity of innovation of developers and infomediaries. According to de la Fuente (2012), impulse of policies and availability of mature technology standards as the semantic web, are enabling a great revolution in the way of distributing and consuming public information. Both, websites and data will link to one another, reducing dramatically the cost of reuse, simplifying their integration with future applications (Berners-Lee, 2009). CTIC Foundation (2011) suggests configuring a kind of ecosystem among government, companies and citizens to promote the re-use of Public Sector Information (PSI) and thus contribute to social welfare (Harrison, Pardo \& Cook, 2012).

Yu \& Robinson (2012) indicate that publish data, in a structured way, is only a necessary condition to dispose of new applications and services, in most cases it is not realistic to expect that innovation occurs automatically.

An action plan is required then, to stimulate the consumption of a dataset between infomediaries companies and developers; this promotes the creation of new applications, driving an economical area whose axles are on one side technical and on the other business. Promoting interoperability and transparency between Public Agencies (PAs) must be a key element of the plan (CTIC, 2011).

Other components to consider in a plan are: gathering information from citizens to know what is the most relevant information to them, incorporating social networks to common channels of participation, since Open Government Data projects (OGD) 
succeed where they satisfy the existent demand of information and commitment (Yu \& Robinson, 2012). In short, there must be an active partnership between government and private stakeholders.

It is necessary to spread out the efforts made by PAs. The plan proposed by CTIC Foundation, incorporates access points (open data portals) and measurement and follow up of the action plan impact. This last point requires establishing a set of indicators that will facilitate measuring and plan targets compliance.

In this context, we give some recommendations to address this complex task, synthetizing in a roadmap the implantation of a sort of OGD ecosystem between government and concerned community (stakeholders). Then, based on this roadmap, the Open Data Implantation Guide (ODIG) provides 15 recommendations. If PAs follow ODIG recommendations, then they will have a maturity level equal to or above 3 from a maximum of 4 in the maturity model shown in Solar et al. (2012).

In Section 2 we show the state of the art in which we base the open data implementation guide shown in Section 3. Finally, we conclude in Section 4.

\section{State of the Art}

Three sources provided information for the development of recommendations tailored to reality. The first one related to the bibliography in OGD subject, from which different proposals of open data implementation arise. The second source, related to the results of the Open Data Maturity Model application, known as OD-MM, from which are collected the recommendations suggested by the model; and the last is a survey carried out by stakeholders and OGD experts around the world.

\subsection{Experiences Reported in the Bibliography}

In the Obama's emblematical memorandum, described in detail in McDermott (2010), the open government directive instructs PAs to include in their plans, linking to a website that has information about their Freedom of Information Act (FOIA) processing and processes. It includes a description of the staffing, organizational structure, agency's capacity to analyze, coordinate, and respond to such requests in a timely manner, and if the PA has a significant backlog, milestones that detail how the agency will reduce its pending backlog of outstanding FOIA requests by at least $10 \%$ each year.

The directive requires executive departments and PAs to take the following steps to the goal of creating a more open government: (1) Publish government information online; (2) Improve the quality of government information; and (3) Create and institutionalize a culture of open government.

Required components of the open government plans developed by PAs are: Transparency; Participation; Collaboration; Flagship initiative; and Public and agency involvement.

In May 2012 they released the digital strategy of the U.S. Federal Government in the document entitled: "Digital Government: Building a 21st Century Platform to 
Better Serve the American People". This document establishes 4 strategic principles that will guide initiatives in digital government, as follows: Focus on information; Shared platform; Focus on the customer; and Security and privacy.

Another case is the government of Australia that provides 13 recommendations as a guide to Government 2.0, summarized in Gruen (2009).

Based on the selected Open Government Initiatives (OGI), relevant literature, and interviews with several PAs, Lee \& Kwak (2011) identify ten key challenges for open government implementation in three dimensions: Organizational; Technology; and Government-wide challenges. Lee \& Kwak (2011) present also 15 recommendations that PAs can use to effectively implementing their OGI.

As measures for a local government (municipal) in ORSI (2010) ten measures are proposed. In the same sense, but in a specific application scope in the U.S., the 8 steps guide in Kaufman \& Wagner (2012), designed for transportation agencies: to open and maintain data, overcome potential obstacles, and create a relationship with users. The steps are: Find your data; Convert data; Test your output; Write up a license agreement; Publish and publicize; Update and modify as needed; and create and maintain a dialogue.

\subsection{Recommendations Collected from OD-MM Maturity Model}

OD-MM maturity model (Solar et al., 2012) applied to six PAs in three countries in Latin America (Chile, Colombia and El Salvador), provided a diagnosis to each PA. Each diagnosis generates its corresponding roadmap with recommendations to continue evolving to the next level of the maturity model. Recommendations to improve on lesser capacity level issues detected in the application of the OD-MM model implementation are typical of a more advanced stage of this new way of governing. It is necessary therefore, to move towards an integrated State, transparent and participatory that solves the problems of citizens and private institutions.

From the recommendations obtained directly from the roadmaps automatically generated by OD-MM model, the following are the suggestions most frequently generated in the implementation of the model:

1 Create training initiatives on issues related to OGD, as the use of IT tools, digital communication systems, office automation, e-services, etc. Create an OGD specific training plan identifying needs of training and other pertinent matters, where staff responsible of OGD training requires an appropriate training.

2 Manage projects with established procedures. Create a Project Management Office (Letavec \& Bolles, 2010), to ensure the compliance of standard procedures in management of all OGD projects of the organization.

3 Establish metrics to assess OGI. Prepare a standard and compliance goals to measure results of programs and initiatives. Create a regular and systematic assessment plan to identify a set of appropriate metrics to evaluate OGI performance, as the compliance of external regulations, among others.

4 Publish numerous open data to the community, covering the entire organization.

5 Create full indicators, with internal tracking. Perform a light analysis of results and propose evident improvement measures. 
6 Offer documents and materials, either of informative or strategic and technical character. Organize workshops with a more technical and/or business delineation.

7 Offer some self-financing opportunity or give adequate information about the possibility of external financing usable in the OGD project development.

\subsection{Expert's Opinion}

Consultations to stakeholders and OGD experts from 15 countries of four continents (America, Asia, Australia and Europe) gave a first-source perception about impacts, either social or economic, that open data have, its eventual reuse, and recommendations to fostering OGDs.

\subsubsection{Survey Methodology}

The methodology consisted primarily in building a poll that could be answered in few minutes, to have a higher rate of responses. Most of the questions were closed inquiries; the only requirement was to mark just the offered alternatives. Finally, the poll had 9 questions from which two were open answers and optional. Built in a Google doc format, this poll provided the chance of answering directly on the web.

The second step was the identification of a group of experts in OGD, for the poll delivery. Those contacted before were favored, since this increased the chances of a response within the time limits.

First three questions try to capture the importance of OGD for the expert, specifically on social and economic impact. Then, it is also important to know their opinion on issues such as the most relevant scopes, costs, benefits, risks and barriers for the OGD implementation. Finally, they responded an open question about their immediate experience regarding to specific results of some impact appreciated, either social, or economic, resultant of OGD and/or its subsequent reuse. An open question remained for further comments to help promoting OGI.

\subsubsection{Survey Results}

Each of the experts selected three areas they thought could create the greatest OGD impacts and its reuse. As shown in Table 1, the areas of "Transport" and "Transparency" are a third of the most named among 15 scopes shown, followed at a distance by the environmental issue.

According to the experts polled, most important benefits or advantages for society of the OGD and its reuse are "Transparency" with $25 \%$, followed closely by increased citizen's "participation" (20\%). It is interesting to emphasize they mentioned, with a significant frequency (14\%), the benefits into PAs reflected in an increased effectiveness and efficiency of public policies. On the other hand, "updated information/knowledge" (rank 8 in Table 2) was an alternative not mentioned in the poll, but included by self-pollees. The economic benefits as "Economic development" and "Entrepreneurship", mentioned together with an $18 \%$, is a significant figure and consistent with the importance level of the economic impact as per the same experts. 
Table 1. Scope of OGD Higher Impacts

\begin{tabular}{clr}
\hline Rank & Scope & \% \\
\hline 1 & Transport & $17 \%$ \\
2 & Transparency & $16 \%$ \\
3 & Environment & $9 \%$ \\
4 & Culture and Recreation & $7 \%$ \\
5 & Public Administration & $7 \%$ \\
6 & Meteorology & $6 \%$ \\
7 & Tourism & $5 \%$ \\
8 & Delinquency & $5 \%$ \\
9 & Education & $5 \%$ \\
10 & Finances & $5 \%$ \\
11 & Health & $4 \%$ \\
12 & Business & $4 \%$ \\
13 & Properties and Land Registry & $4 \%$ \\
14 & Political scope & $4 \%$ \\
15 & Scientific & $2 \%$ \\
\hline & Total & $\mathbf{1 0 0 \%}$ \\
\cline { 2 - 3 }
\end{tabular}

Table 2. OGD Benefits

\begin{tabular}{clc}
\hline Rank & Benefit & \% \\
\hline 1 & Transparency & $25 \%$ \\
2 & Participation & $20 \%$ \\
3 & Trust & $17 \%$ \\
4 & Efficiency and effectiveness of public policies & $14 \%$ \\
5 & Economic development & $12 \%$ \\
6 & Entrepreneurship & $6 \%$ \\
7 & Quality of life & $3 \%$ \\
8 & Updated information/knowledge & $3 \%$ \\
\hline & Total & $\mathbf{1 0 0 \%}$ \\
\cline { 2 - 2 }
\end{tabular}

Table 3 presents main barriers or difficulties in initiating open data projects. As shown, the "Lack of political will" is the most frequently mentioned factor that avoids the implantation of OGI. Second, and with similar percentages (16\% each), are the "cultural" factors, as well as the "lack of appropriate laws", and "lack of leadership" (very similar to the "Lack of political will").

Table 4 presents the responses associated to risks, limitations and costs related to OGD in general. It is remarkable that the issue "Sensitive data" appears as the main constraint or risk associated with OGI. Second, "Processing costs" of large volumes of data; and far behind in third place is the "investment" associated.

Some comments given by the experts are hereunder detailed:

- Difficulties in impacts measuring; there is a consensus about existent impacts, but for example, measuring the increase of trust in PAs does not seem easy. Other impacts associated with private benefits are measurable, but difficult to capture.

- In relation to the previous point, impacts in PA are also mentioned, by improving internal processes. This with respect to organization and classification of information, to make it more accessible. Additionally, fostering innovation in the country is not a minor issue. 
- More training and divulgation: Several comments point out to the need of sensitizing authorities with seminars, divulgation and training campaigns for OGD issues.

- Finally, there are other comments emphasizing the urgency of these issues and the need to accelerate data opening processes, otherwise public pressure will become stronger increasing the discredit of government institutions.

Table 3. Barrier for OGD Implantation

\begin{tabular}{llc}
\hline Rank & Barriers & \% \\
\hline 1 & Lack of political will & $20 \%$ \\
2 & Cultural problems & $16 \%$ \\
3 & Lack of laws and regulations & $16 \%$ \\
4 & Lack of leadership & $14 \%$ \\
5 & Lack of qualified personnel & $12 \%$ \\
6 & Ignorance & $12 \%$ \\
7 & Lack of confidence & $8 \%$ \\
8 & Very high costs & $0 \%$ \\
\hline & Total & $\mathbf{1 0 0 \%}$ \\
\hline
\end{tabular}

Table 4. OGD Costs and Limitations

\begin{tabular}{ll}
\hline Costs & $\mathbf{\%}$ \\
\hline Sensible data & $39 \%$ \\
Processing cost & $21 \%$ \\
High investments & $14 \%$ \\
None & $11 \%$ \\
Publication of useless data / data quality is poor & $7 \%$ \\
Data not reused & $4 \%$ \\
Regulation & $4 \%$ \\
Citizen's fear & $0 \%$ \\
\hline Total & $\mathbf{1 0 0 \%}$ \\
\hline
\end{tabular}

\subsection{Comparing Proposals}

When comparing the recommendations of the Australian Government (Gruen, 2009), with the U.S. government (McDermott, 2010), and ORSI (2010) for local governments as municipalities, private proposal of Lee \& Kwak (2011), and an implementation proposal in a specific scope of application such as transport (Kaufman \& Wagner, 2012), it is possible to find common factors to all of them.

First, in all proposals is mentioned, as an important action "A declaration of open government by the government", or else, they recommend "Develop and communicate a government-wide strategy", like the Open Government Directives (McDermott, 2010). In the same way, "coordinate with leadership, guidance and support" is similar to "align OGI with the agency's goals" or "institutionalize OGI" of Lee \& Kwak (2011), and to "create and institutionalize a culture of OGD" in McDermott (2010).

The Australian recommendation: "Make PSI open, accessible and reusable" is not different to "Public data opening ", from ORSI, or "consider conducting pilot projects 
and/or establishing centers for excellence" from Lee \& Kwak (2011), or "flagship initiatives" in Obamas' memorandum. Just as "Find your data; Convert data; and Test your output" is only the way to operationalize this.

The proposal "Encourage public servants to engage online" (Gruen, 2009), is not different from the proposals "using tools of internal collaborative work" and "encourage internal participation in the city council" in ORSI (2010), and is similar to "integrate public engagement applications" (Lee \& Kwak, 2011).

Related to the "accessibility" issue, we have "create and maintain a dialogue" in Kaufman \& Wagner (2012), "platforms of participation and citizen's collaboration" in ORSI (2010), and "use a democratic, bottom-up approach" in Lee \& Kwak (2011).

\section{Open Data Implementation Guide (ODIG)}

The following are some of the principles and criteria considered for the ODIG design:

- Simplicity: Time of implementation should not be too extensive, for example, if some laws are not required, initial results should be available within 18 months.

- Quick-Win: This principle means a quick initial development with some visible results that could help legitimizing the initiative and obtaining additional support from stakeholders. This involves the need of a subsequent long-term development, but experience acquired at the beginnings and its legitimization can facilitate the concretion of the following steps.

- Maturity Level: As a result of the above mentioned, this guide intends to provide the first steps towards the development of OGD in a PA. Still, if this ODIG can be properly implemented, it can be guaranteed that, submitted to the OD-MM maturity model (Solar et al., 2012), the entity could achieve a level 3 of maturity or very close to it (for a maximum of 4).

- Share Experience: Since development of OGD is a recent issue in the world (about four years), it is always worth to consider the learning and exchange of experiences between countries and institutions to move forward.

- Data Liberation: The data considered in ODIG are those contained and recorded in any format; they are collected, produced, and/or received by PAs, and should be available to the public in accordance with Harrison et al. (2012) recommendations.

ODIG is a consequence of the OD-MM application since it incorporates elements detected as weak in pilot PAs; as well as elements of both, bibliographic exploration and field research carried out throughout firsthand sources.

For the ODIG development and organization, the roadmap displayed in the following section, is a reference conceptualized with OD-MM maturity model domains. For this reason, the ODIG classified three groups, corresponding to the three OD-MM model domains: the first is the "Institutional and Legal Perspective" including eight recommendations concerning organizational and management issues. Second, the "Technological Perspective" with two recommendations, and finally the "Citizen's and Business Perspective" incorporating 5 recommendations on issues related to data reuse, by the concerned community. 
Nevertheless, and following the principle of simplicity already mentioned, ODIG develops the technological domain in a simplified way focusing only on fundamental issues addressed to decision makers, trying to avoid technicalities that could obstruct its comprehension. The reasons of this are: (1) Technology is by no means the main reason in OGD, since technology is just a means used to achieve it (Calderon \& Lorenzo, 2010), and (2) Technology is in a constant change, it repeatedly surprises the experts themselves; therefore, the risk is high when offering detailed technology standards that in short term will be obsolete.

\subsection{A Roadmap}

Following are the general guidelines, called roadmap, oriented to the formulation of the ODIG. The order of this roadmap is only referential; it does not pretend to be exhaustive, not either is it necessary to complete all steps in a more developed level, and it can certainly perform some tasks in parallel:

1 Have an organization appropriate to build OGD that should not be necessarily equal to the existent, for the management of traditional e-government activities.

2 Hire and generate a training plan to provide qualified professionals in OGD.

3 Articulate an institutional statement provided by the Presidency in favor of an open government, as soon as possible, which should be part of the objectives definition and the strategy to follow on this topic (de la Fuente, 2012).

4 Have an interoperability platform between different PAs (desirable).

5 Datasets opening. Prior to this, carry out a market research regarding to the most relevant and priority data that companies and citizenship in general are requiring.

6 Develop an OGD policy including the adoption of standard open formats for data and metadata, to facilitate its later reuse.

7 Construction of an official website of OGD that includes the results of a previous study following the best international practices in the field.

8 Establish an action plan to stimulate data consumption between companies and especially among infomediaries.

9 Create alliances and agreements with stakeholders from civil society and private sector to promote specific projects of data reuse of a public value for citizens and/or PAs. Harrison et al. (2011) proposes alternatively that planning and assessing OGD being addressed within a "public value" framework.

10 Establish a measurement of initial diagnosis of PAs maturity level regarding to OGD, to serve as a baseline in the periodic measurement of progresses expected and approaches, and to facilitate necessary corrective and timely decision making.

This Decalogue is presented in generic or added terms; therefore, it represents the general framework and starting point for developing ODIG points for governments. 


\subsection{Fifteen Recommendations of ODIG}

Considering a first source expert opinion, plus bibliographic research and elements development, in each of the two not technological perspectives of the open data maturity model, there is a sequence and prioritization of steps that the executor must consider when implementing ODIG. The estimated horizon of time considered ranges from 18 months and two years, depending on the starting point in each case:

1. THE EXISTENCE OF AN INSTITUTIONAL FRAMEWORK WITH A RECOGNIZED ORGANIZATION FOR OGD: This is the starting point to implement an OGI with some probability of success sustained over time. It might be ascribed to an existing e-government initiative or other related organization. This institutional framework must generate an organizational structure where formally defined positions and proficiencies cover the areas of management, planning and technical. For example, it is desirable to have a person in charge of PSI re-use (known as PSI manager), a contents manager who knows the procedures for data processing, with knowledge of databases and their applications, and web portals. Finally, a systems manager with competences in the equipment catalog and IT systems able to support storage and data publishing.

2. EXISTENCE OF A RECOGNIZED LEADER IN CHARGE OF IMPLEMENTING AN OGD INITIATIVE: It is necessary to appoint a suitable and sufficiently empowered person to hold a position that requires not only technical skills, but also a good political management to interact with different social levels of the public sector, and organizations of the civil world. This person should be responsible for developing a strategy, and drive the implementation process.

3. FORMULATION OF AN OGD STRATEGIC DEVELOPMENT PLAN: When formulating a plan, the recommendation is to involve different social actors and at diverse instances; namely, to generate seminars inviting diverse social organizations to make their contributions; in parallel, leave enough room on the website for the citizen feedback. Although the process seems slower, in the long-term will generate greater legitimacy. Additionally, it is worth to establish first bonds with civil society, actors of an OGI. Furthermore, the advice is to provide activities to the short, medium and long term plan. Short term measures, as far as possible, must be visible enough to generate a positive impact on the population and thus, more support for long term actions. This approach must incorporate a communication strategy.

4. CONSTRUCTION AND DELIVERY OF NECESSARY LAWS TO THE CONGRESS FOR A BETTER OPERATION OF THE OGD: This activity may vary from country to country and in some cases, omitted; however, in general it is necessary to promulgate laws related to the regulation of transparency in information and protection of sensitive information. OGD supervisor must seek legal advice to achieve the development of these laws, and promote a flexible remittance and promulgation of them. After this promulgation of laws, training and divulgation of them is the next step among interested stakeholders for a better understanding/interpretation of their scopes. 
5. PROMULGATION OF POLICIES AND INTERNAL REGULATIONS: Not only standards are important (i.e., those related to information management procedures and their conversion to standard formats), but to keep evidence of their compliance through various control mechanisms. Additionally, the pertinent authority must ensure a proper understanding of the standards. An issue related to this point is the formulation of an open data policy including the adoption of open standards formats for data and metadata to facilitate their later reuse.

6. TRAINING PLAN IN OGD: Several studies inferred that it is essential to have support of trained personnel, which in general has not been formally resolved. Some institutions consider that an 'on the way' learning is enough, or that more advanced professionals in charge should be able to solve problems. Experience shows that this long-term strategy is inadequate and may increase the costs. The point again, is to follow the plan. This involves that after about six months, there will be available a significant number of key personnel trained in the techniques of OGD, digital communication systems, IT tools, e-services, etc.

7. Project Management OfFice Development: The OGD implementation requires the development of several projects; therefore, it is necessary to ensure the compliance of standard procedures in management of OGD projects. This is a weakness detected in the field of software engineering; it tells of a high percentage of IT project failures, or at least backlogs in the compliance of delineated goals.

8. HAVE A PERFORMANCE ASSESSMENT SYSTEM OF THE PROJECTS: In general, it is possible that this system already exists in the PA. However, the results of documented experiences indicate that often no formal metrics mechanisms are present to measure projects performance, neither the establishment of specific goals.

9. DEVELOPMENT OF A STUDY OF REQUIRED ICT INFRASTRUCTURE CAPACITY: Decision makers must be aware of the need of safeguarding that systems will have sufficient capacity to manage the demands and requirements of citizens and infomediaries companies. (i.e., avoid equipment's saturation with web services).

10. GRADUALLY INCORPORATE SEMANTIC TECHNOLOGIES: These technologies are available today and it is a need to incorporate them, although their use is initially on an experimental basis, to train technical staff of the PA. With this, the PA can easily reach levels 4 and 5 stars of Berners-Lee (2009). The use of these technologies also allows to better manage a multiplicity of catalogs of different sources, from both, national and local governments; also from other State authorities, private sources, etc.; this, if the option is a distributed model of catalogs. In other words, facilitates interoperability, data aggregation, and catalogs, plus its updating from external sources.

11. DEVELOPMENT OF A FIRST OGI: This first initiative, recommended as a pilot, must be the most emblematic and with significant impact in the short term. Identification of the most relevant information and at the same time easier to open will be a tool for each country; however, it is important to consider citizens' participation in 
the development of this initiative, in at least some of its stages. We suggest identifying those less sensitive data categories, but with a high impact when applying Quick-win criterion. This will avoid controversies and complexity that could delay the project.

12. EXISTENCE AND MANAGEMENT OF DATASETS INDICATORS FOR ACCESS AND/OR DOWNLOADING, TOGETHER WITH DATA MONITORING: Monitoring of access and data downloading is essential to assess the level of success, especially of the first initiative. This allows taking appropriate corrective actions. It is highly recommended to complement it with periodic public opinion polls (re-users).

13. PROMOTION ACTIONS FOR RE-USE: Offering of various documents and materials, both informative and of strategic and technical character encourage the use of OGD. Speeches, workshops, seminars, applications contests, are some of the initiatives that government must have available in a systematically, to support developers and data reuse. Likewise, the recommendation is to publish successful stories, of great impact in the portal site, with metrics that establish the benefits and impacts that they had for users if possible.

14. EXISTENCE OF A CHANNEL FOR COMPLAINTS AND CONFLICTS RESOLUTION: The portal must have at least a form available with clear instructions, helpful to canalize developers and users difficulties with data reuse. This mechanism will be essential to improve aspects related to the reuse.

15. EXISTENCE OF A FORMAL CHANNEL OF PARTICIPATION AND COLLABORATION OF CIVIL SOCIETY: As mentioned above, participation and cooperation of citizens should be the cornerstone of any OGD project to ensure its success. Procedures and verification of opinions and suggestions must be available for consultation before future improvements. Opinions should have a rating system (public vote).

\section{Conclusions}

One of the recurrent elements in the bibliography is the one related to political leadership, cited as critical to a successful OGD implementation. It is an element present in all the proposals of OGD implementation plans. This element also emerges as one of the weaknesses found in the OD-MM maturity model application, and all OGD experts polled emphasize it as well, so its presence is natural in the ODIG.

The new elements in the actions proposed by the ODIG are the formulation of OGD training plans, the formulation by the PA of a strategic OGD development plan, and having a performance evaluation system of OGD projects, including the development of a PMO.

The experience of applying a pilot to six PAs in three Latin American countries, demonstrates that the presented ODIG, assumes its weaknesses detected in the diagnosis of these PAs. Therefore, when following the actions proposed by ODIG, these PAs will reach level 3 of maturity, or very close to it (from a maximum of 4), for sure. 
Acknowledgements. The authors would like to thank Gonzalo Valdes, Gastón Concha, Cristián Torres, and José Gleiser for their work in the project. This work was partially supported by the grants DGIP 241142, International Development Research Center (IDRC/CDRI) with the collaboration of Inter-american Organization for Higher Education (OUI).

\section{References}

1. Berners-Lee, T.: Linked Open Data. What is the idea? (2009), http: / /www. thenationaldialogue.org/ideas/linked-open-data

2. Calderón, C., Lorenzo, S.: Open Government. Algón Editores (2010)

3. CTIC, World Wide Web Foundation and CTIC. Open Government Data, Feasibility Study in Chile (2011), http://public.webfoundation.org/2011/05/OGD_Chile. pdf

4. de la Fuente, C.: Gobierno como Plataforma: Retos y Oportunidades. En: "El desafio hacia el gobierno abierto en la hora de la igualdad". CEPAL - Colección Documentos de proyectos (2012), http://www.eclac.cl/ddpe/publicaciones/xml/9/ $46119 / \mathrm{w} 465 \cdot \mathrm{pdf}$

5. Gruen, N.: Engage: Getting on with Government 2.0 (2009), http: / /www. finance.gov.au/publications/gov20taskforcereport/ doc/Government20TaskforceReport.pdf

6. Harrison, T.M., Guerrero, S., Burke, G.B., Cook, M., Cresswell, A., Helbig, N., Hrdinová, J., Pardo, T.: Open Government and E-Government: Democratic Challenges from a Public Value Perspective. In: Proc. of the 12th Annual Int. Conf. on Digital Government Research, College Park, MD, USA (June 2011)

7. Harrison, T.M., Pardo, T.A., Cook, M.: Creating Open Government Ecosystems: A Research and Development Agenda. Future Internet 4, 900-928 (2012)

8. Kaufman, S.M., Wagner, R.F.: Getting Started with Open Data: A Guide for Transportation Agencies. NYU Rudin Center for Transportation Policy and Management (2012)

9. Lee, G., Kwak, Y.H.: An Open Government Implementation Model: Moving to Increased Public Engagement. IBM Center for the Business of Government (2011)

10. Letavec, C., Bolles, D.: The PMOSIG Program Management Office Handbook. J. Ross Publ., 464 pages (2010)

11. McDermott, P.: Building open government. Government Information Quarterly 27, 401-413 (2010)

12. ORSI, Observatorio Regional de la Sociedad de la Información de Castilla y León (ORSI). Open Government. 10 ideas para hacer tu ayuntamiento abierto (2010)

13. Solar, M., Concha, G., Meijueiro, L.: A Model to Assess Open Government Data in Public Agencies. In: Scholl, H.J., Janssen, M., Wimmer, M.A., Moe, C.E., Flak, L.S. (eds.) EGOV 2012. LNCS, vol. 7443, pp. 210-221. Springer, Heidelberg (2012)

14. Yu, H., Robinson, D.G.: The New Ambiguity of 'Open Government' 59 UCLA L. Rev. Disc. 178 (December 12, 2012), http: //ssrn. com/abstract $=2012489$ 